\title{
Pyogene Ventrikulitis als Komplikation bakterieller Meningitiden
}

\section{Pyogenic Ventriculitis as a Complication of Bacterial Meningitis}

\author{
Autoren \\ L. Kraayvanger, R. Weber, P. Berlit \\ Institut \\ Klinik für Neurologie, Alfried Krupp Krankenhaus, Essen
}

\author{
Schlüsselwörter \\ - Ventrikulitis \\ - Meningitis \\ $\checkmark$ pyogen \\ Keywords \\ ventriculitis \\ - meningitis \\ $\checkmark$ pyogenic
}

Bibliografie

DOI http://dx.doi.org/

$10.1055 / \mathrm{s}-0034-1396844$

Akt Neurol 2015; 42: 43-45

(c) Georg Thieme Verlag KG

Stuttgart · New York

ISSN 0302-4350

Korrespondenzadresse

Laura Kraayvanger

Klinik für Neurologie

Alfried Krupp Krankenhaus

Alfried-Krupp-Straße 21

45131 Essen

laura.kraayvanger@krupp-

krankenhaus.de

\section{Zusammenfassung}

$\nabla$

Pyogene Ventrikulitiden sind eine seltene und potentiell schwer verlaufende Komplikation intrakranieller Infektionen. Häufig sind sie die Ursache schwierig zu eradizierender Keime. Aufgrund untypischer klinischer Präsentation kann die Diagnose erschwert sein. In der Bildgebung ist die MRT der CT überlegen. Als wesentlicher Befund in der MRT findet sich eine Signalsteigerung in den Hinterhörnern der Seitenventrikel in den diffusionsgewichteten Sequenzen als Ausdruck des Empyems. Wir präsentieren 2 Kasuistiken einer bakteriellen Meningitis mit pyogenem Ventrikulitis-Nachweis im CCT und cMRT, davon einen Fall mit initial ungewöhnlichen klinischen und Liquorbefunden, und geben einen aktuellen Literaturüberblick.

\section{Einleitung}

$\nabla$

Pyogene Ventrikulitiden sind eine seltene Komplikation intrakranieller Infektionen, die eine Ansammlung eitriger Flüssigkeit im Ventrikelsystem aufweisen. Als synonyme Begriffe werden häufig Ventrikelempyem, Pyocephalus oder Ependymitis verwandt. Häufige Ursachen einer pyogenen Ventrikulitis sind vorangegangene invasive diagnostische oder therapeutische Prozeduren (z.B. die Anlage eines Liquorshuntes oder Lumbalpunktionen), Rupturen intrakranieller Abszesse oder seltener die direkte Ausbreitung einer bakteriellen Meningitis, deren Erreger schwierig zu eradizieren sind. Klinisch können sie mit geringen oder atypischen Symptomen einhergehen und daher schwierig zu diagnostizieren sein, bei nicht rechtzeitiger Erkennung aber dennoch tödlich verlaufen. Eine zerebrale Bildgebung kann in der Diagnosestellung hilfreich sein, wobei nur wenige Berichte über kraniale CT- und MRT-Befunde bei erwachsenen Patienten mit pyogenen Ventrikulitiden existieren.

\section{Abstract \\ $\nabla$}

Pyogenic ventriculitis is a rare complication of intracranial infections with possibly fatal outcome. Frequently, it is caused by persistent infection with pathogens that are difficult to eradicate. Its symptoms may be subtle and therefore cause delay of correct diagnosis. A common finding in MR imaging is the detection of pyogenic debris by markedly increased signal intensity on diffusion-weighted images in the occipital horn in the lateral ventricles. We present 2 cases of bacterial meningitis with pyogenic ventriculitis, one with initially unusual clinical presentation and laboratory findings along with a survey of current literature.
Wir berichten über 2 Meningitis-Patienten, bei denen eine pyogene Ventrikulitis mithilfe von bildgebenden Verfahren diagnostiziert wurde und geben eine aktuelle Literaturübersicht $\mathrm{zu}$ dieser seltenen Komplikation.

\section{Kasuistik}

Fall 1

Eine 73-jährige Frau stellte sich aufgrund von starken Kopfschmerzen in unserer Notaufnahme vor. 3 Tage zuvor war eine zervikale und lumbale Infiltrationsbehandlung erfolgt. In der Nacht vor der stationären Aufnahme war sie im häuslichen Umfeld rezidivierend gestürzt. Klinisch zeigte sich ein ausgeprägter Meningismus, darüber hinaus jedoch kein fokal neurologisches Defizit. Während des stationären Aufenthaltes entwickelte sie Fieber. Laborchemisch bestanden eine

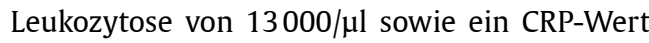
von $17,4 \mathrm{mg} / \mathrm{dl}$ bei normwertigem Procalcitonin. Liquordiagnostisch zeigte sich der typische 
Befund einer bakteriellen Meningitis mit einer Zellzahl von $10789 / \mu l$, einem Gesamtprotein von 693,4 mg/dl, einem erhöhten Lactatwert von $10 \mathrm{mmol} / \mathrm{l}$ und einem deutlich erniedrigtem Glukosewert von $6 \mathrm{mg} / \mathrm{dl}$. CT-morphologisch fand sich eine hyperdense Spiegelbildung im Hinterhorn des linken Seitenventrikels ( $\bullet$ Abb. 1). Hals-Nasen-Ohrenärztlich zeigte sich ein Normalbefund, der Urinstatus war unauffällig, das Röntgenbild des Thorax zeigte eine Belüftungsstörung des linken Unterlappens, welche differenzialdiagnostisch als beginnendes Infiltrat gedeutet wurde. Ein Erregernachweis gelang in den Blut- sowie Liquorkulturen nicht, auch das Grampräparat zeigte sich negativ. Die Patientin wurde intravenös antibiotisch mit Ceftriaxon über 10 Tage behandelt. Eine Kontrollpunktion am zehnten Behandlungstag zeigte $119 / \mu \mathrm{l}$ Zellen, einen Proteinwert von $822 \mathrm{mg} / \mathrm{l}$, einen Lactatwert von $4,4 \mathrm{mmol} / \mathrm{l}$ und normale Glukosewerte. Aufgrund des guten klinischen Ansprechens der antibiotischen Behandlung innerhalb der ersten 48 Stunden wurde auf eine weitergehende spinale Bildgebung nach der Infiltrationsbehandlung verzichtet. Ein lokaler spinaler oder paraspinaler Klopfschmerz fand sich nicht. Nach 2 Wochen wurde die Patientin beschwerdefrei in das häusliche Umfeld entlassen.

\section{Fall 2}

Ein 86-jähriger Mann wurde aufgrund einer Ungeschicklichkeit der rechten Hand sowie eines Hängenbleibens am Boden mit dem rechten Bein während des Gehens aus einer internistischen Klinik mit dem Verdacht auf Schlaganfall zugewiesen. Bei Aufnahme bestand kein fokal neurologisches Defizit mehr. 6 Stunden nach der Übernahme entwickelte der Patient allerdings eine hochgradige motorische Aphasie, eine rechtsseitige Hemiparese sowie Fieber bis $39^{\circ} \mathrm{C}$. Laborchemisch zeigte sich eine Leukozy-

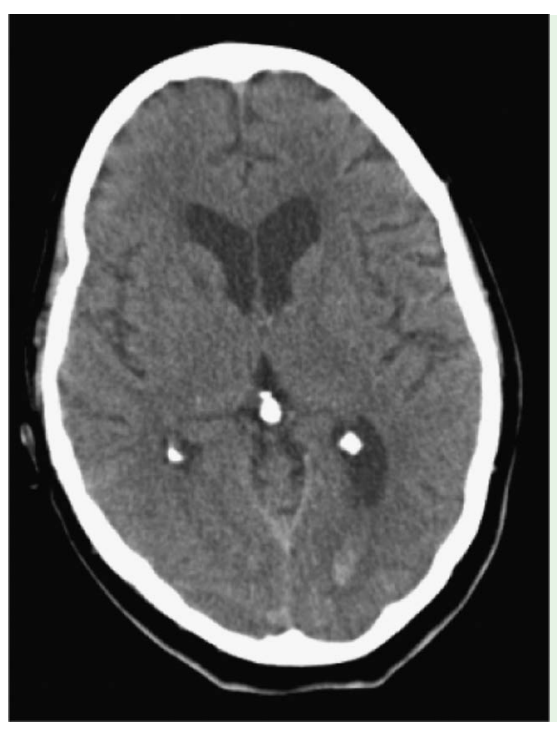

Abb. 1 Kraniales CT mit hyperdenser Spiegelbildung im Hinterhorn des linken Seitenventrikels. Die Abbildung wurde mit freundlicher Genehmigung der Klinik für Radiologie und Neuroradiologie, Alfried Krupp Krankenhaus Essen, zur Verfügung gestellt.
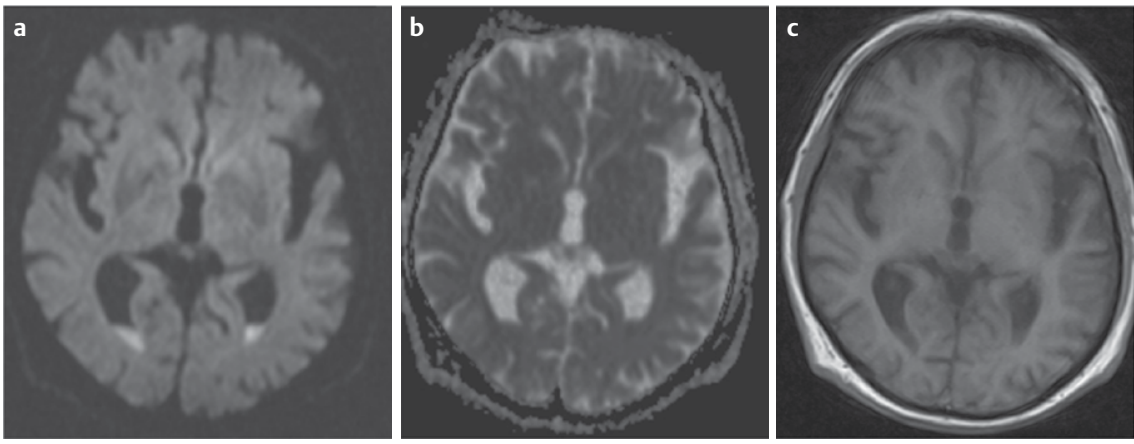

Abb. 2 Kraniales MRT mit sedimentierter Signalalteration in den diffusionsgewichteten Aufnahmen a, korrespondierendem Signalabfall im ADC-Map b und leicht hyperintensem Signalverhalten in der T1-Sequenz c. Die Abbildung wurde mit freundlicher Genehmigung der Klinik für Radiologie und Neuroradiologie, Alfried Krupp Krankenhaus Essen, zur Verfügung gestellt. 
auftreten, wie Zytomegalieviren oder Nocardien [1]. Bildgebende Verfahren können bei der Diagnose wichtige Hinweise liefern, obwohl bisher nur wenige Berichte über kraniale CT- und MRT-Befunde bei erwachsenen Patienten mit Ventrikulitiden veröffentlicht wurden. Häufigster Befund ist der Nachweis eines ventrikulären Zelldebris in $94 \%$ der Fälle. Des Weiteren können häufig ein Hydrocephalus, periventrikuläre hyperintense Signale in der FLAIR-Sequenz der MRT-Bildgebung oder ein ependymales Kontrastmittelenhancement nachgewiesen werden [2,3]. Bei den geschilderten Fällen konnten bis auf den Zelldebris keine der anderen Auffälligkeiten nachgewiesen werden, wobei Aussagen zum Kontrastmittelverhalten des Ependyms bei keinem der Patienten getroffen werden können, da die erste Patientin keine MRT-Untersuchung erhielt und beim zweiten Patienten keine Kontrastmittelapplikation erfolgte. Der Zelldebris findet sich aufgrund der Lageabhängigkeit am häufigsten in den Hinterhörnern der Seitenventrikel und imponiert leicht hyperintens in den T1-gewichteten und leicht hypointens in den T2-gewichteten Aufnahmen. Bedingt durch die hohe Viskosität der eitrigen Flüssigkeit mit Hyperzellularität sowie Wasserbindung an den Makromolekülen fällt der Zelldebris - ähnlich wie ein intrakranieller Abszess - durch ein hyperintenses Signal in den diffusionsgewichteten Sequenzen mit entsprechendem Signalabfall in den ADC-Aufnahmen als Ausdruck einer eingeschränkten Protonendiffusion auf [4]. Diese intraventrikulären Signalveränderungen fanden sich in einer rezenten retrospektiven Studie bei $54,7 \%$ der Patienten mit bakterieller Meningitis, die eine kraniale MRT erhielten und damit deutlich häufiger als in älteren Studien [6]. Procalcitonin im Serum kann hilfreich in der Abgrenzung einer bakteriellen von einer andersartigen Meningitis bzw. Ventrikulitis sein. Spezifische Liquorparameter sind hierfür nicht etabliert [1,5].

Der erste Fall der 73-jährigen Patientin zeigte typische klinische und liquordiagnostische Befunde, die rasch die Diagnose einer bakteriellen Meningitis nahelegten. CT-morphologisch wurde die pyogene Ventrikulitis inzidentell als Begleitbefund nachgewiesen. Anamnestisch lag hierfür mit den vorangegangenen zervikalen und lumbalen Infiltrationen ein typischer Provokationsfaktor vor. Wir gingen trotz des fehlenden Keimnachweises und des bestehenden Infiltrates im Röntgenbild des Thorax am ehesten von den vorangehenden infiltrierenden Eingriffen als Eintrittspforte und Ursache der Meningitis bzw. Ventrikulitis aus. Im zweiten Fall des 86-jährigen Mannes war die zur Aufnahme führende klinische Symptomatik zunächst als fokale zerebrale Ischämie gedeutet worden. Liquordiagnostisch zeigte sich nach Auffieberung mit einer Zellzahl von $6 / \mu$ l bei normwertigen Glukose- und Lactatwerten eingangs ebenfalls ein für eine bakterielle Meningitis untypischer Befund. Hierbei handelte es sich am ehesten um einen sog. Status bacillosus, d.h. eine hohe Bakteriendichte im Liquor bei niedriger Zellzahl, der einen ungünstigen Verlauf anzeigen kann. In diesem Fall erbrachte erst die am dritten Behandlungstag durchgeführte MRT-Untersuchung die typischen bildgebenden Befunde einer Ventrikulitis bei fehlendem Nachweis einer Ischämie. Die in der Blutkultur und im Liquor nachgewiesenen oralen Streptokokken sind eher ungewöhnliche Erreger einer bakteriellen Meningitis oder Ventrikulitis, da sie zur normalen menschlichen Keimflora gehören und per se nur gering pathogen sind. Als Auslöser von Meningitiden nach spinalen Eingriffen wie einer Anästhesie oder Lumbalpunktion (die bei unserem zweiten Patienten aber nicht durchgeführt worden waren) machen sie jedoch bis zu $60 \%$ der Fälle aus. Hierbei konnten viele der Fälle zur oralen kommensalen Flora eines medizinischen Angestellten, der an einer invasiven Prozedur des Patienten beteiligt war, zurückverfolgt werden. Dies belegt nochmals nachdrücklich den Stellenwert einer strikt aseptischen Liquorpunktion sowie der Verwendung eines Mundschutzes gemäß den Leitlinien $[7,8]$. Weitere Risikofaktoren können Komorbiditäten sowie potentiell immunkompromittierende $\mathrm{Er}$ krankungen oder Konditionen wie hohes Alter, Diabetes mellitus, Alkoholismus, sanierungsbedürftiger Zahnstatus, Malignome, Endokarditiden oder Sinusitiden sein $[9,10]$. Einschränkend muss erwähnt werden, dass eine genauere Keimdifferenzierung, welche Hinweise auf den zugrunde liegenden Fokus hätte bieten können, nicht durchgeführt wurde. Der genaue Fokus blieb bei dem Patienten daher ungeklärt, bis auf das hohe Alter ließen sich keine Risikofaktoren eruieren.

In unseren Fällen sprachen beide Patienten gut auf die intravenöse Antibiotikagabe an, häufig können Ventrikulitiden allerdings auch die Ursache einer therapierefraktären Meningitis sein. Ihr Nachweis in den bildgebenden Untersuchungen rechtfertigt dann den Einsatz einer intraventrikulären Antibiose über ein Ommaya Reservoir als Therapieoption.

\section{Fazit für die Praxis}

Pyogene Ventrikulitiden sind seltene und potentiell tödliche Komplikationen intrakranieller Infektionen und können deren Eradikation erschweren. Ihr Nachweis kann über bildgebende Verfahren gelingen. Hierbei ist einer MRT-Untersuchung aufgrund der Möglichkeit, Flüssigkeit in verschiedenen Sequenzen genauer zu differenzieren, der Vorzug zu geben. Signalanhebungen in den Hinterhörnern der Seitenventrikel in den diffusionsgewichteten Aufnahmen sind hierbei ein typischer Befund.

\section{Interessenkonflikt}

$\nabla$

Die Autoren geben an, dass kein Interessenkonflikt besteht.

\section{Literatur}

1 Yuen AHC, Mok FCK. Ventriculitis: an unusual cause of sepsis in an elderly patient who presented with persistent fever. Asian J Gerontol Geriatr 2006; 1: 49-52

2 Kastrup 0 , Wanke I, Maschke M. Neuroimaging of Infections. NeuroRx 2005; 2: 324-332

3 Fukui MB, Williams RL, Mudigonda S. CT and MRI Imaging Features of Pyogenic Ventriculitis. Am J Neuroradiol 2001; 22: 1510-1516

4 Pezzullo J, Tung GA, Mudigonda $S$ et al. Imaging of Pyogenic Ventriculitis. Am J Roentgenol 2003; 180: 71-75

5 Casado Morales MI, Moreno Alonso F, Juárez Belaunde AL et al. Ability of procalcitonin to predict bacterial meningitis in the emergency department. Neurologia 2014, doi:10.1016/j.nrl.2014.07.003

6 Lummel N, Koch M, Klein M et al. Spectrum and Prevalence of Pathological Intracranial Magnetic Resonance Imaging Findings in Acute Bacterial Meningitis. Clin Neuroradiol 2014 Sep 23 Epub ahead of print

7 Wilson M, Martin R, Walk ST et al. Clinical and Laboratory Features of Streptococcus salivarius Meningitis: A Case Report and Literature Review. Clin Med Res 2012; 1: 15-25

8 Diener HC, Weimar C, Berlit P et al., Hrsg. Leitlinien für Diagnostik und Therapie in der Neurologie. 5. vollst. überarb. Aufl. Stuttgart: Thieme, 2012 Kap. 84

9 Kutlu SS, Sacar S, Cevahir N et al. Community-acquired Streptococcus mitis meningitis: a case report. ISRN Infect Dis 2008; 12: e107-e109

10 Fukushima K, Noda M, Saito $Y$ et al. Streptococcus sanguis Meningitis: Report of a Case and Review of the Literature. Intern Med 2012; 51: 3073-3076 